\title{
Décès attribuables à des blessures au Canada en 2015
}

\author{
Xiaoquan Yao, M. Sc.; Robin Skinner, M. Ps.; Steven McFaull, M. Sc.; Wendy Thompson, M. Sc.
}

Diffuser cet article sur Twitter

\begin{abstract}
Résumé
Les blessures demeurent un sujet de préoccupation pour la santé publique au Canada. Disposer de données nationales sur les décès attribuables à des blessures est indispensable pour comprendre l'importance et le profil des blessures. Nous avons utilisé pour cet article la base de données sur les décès des statistiques de l'état civil pour examiner les décès associés aux blessures en 2015. Nous avons réparti les blessures par cause de décès et nous avons effectué une analyse plus approfondie par catégorie de blessure en fonction du sexe et de l'âge. Les blessures non intentionnelles étaient à la sixième place des causes de décès en général, ce classement variant selon le sexe. Les principales causes de décès attribuables à des blessures non intentionnelles étaient les chutes, les empoisonnements, les accidents de la route et la suffocation, avec des variations selon les groupes d'âge.
\end{abstract}

Mots clés : mortalité, causes principales, blessures, blessures non intentionnelles, suicide, chutes, empoisonnements, accidents de la route

\section{Introduction}

Une blessure est un transfert d'énergie à l'être humain à des taux et en quantités supérieurs ou inférieurs au seuil de tolérance des tissus humains. L'importance de la concentration d'énergie hors des bandes de tolérance des tissus détermine la gravité de la lésion ${ }^{1}$. Les causes externes sont les chutes, les accidents de la route, l'empoisonnement, la suffocation, la noyade, le feu, les heurts par ou contre un objet et l'ensemble des autres cas $^{2}$. Les blessures sont classées soit comme non intentionnelles, soit comme intentionnelles : les blessures non intentionnelles se produisent sans qu'il y ait intention de causer un préjudice (par exemple lorsqu'une personne est blessée à la suite d'une chute, d'une brûlure ou d'un accident de la route $^{2}$ ) alors que les blessures intentionnelles résultent d'un acte délibéré de préjudice envers soi-même ou envers autrui (par exemple lors d'un suicide ou d'un homicide $^{2}$ ). Lorsque l'intention n'est pas claire, on classe la blessure comme « à intention non déterminée ».
Les blessures constituent un problème de santé publique tant à l'échelle mondiale qu'au Canada. Près de 5 millions de personnes meurent en effet chaque année dans le monde à la suite de blessures, ce qui correspond à $9 \%$ des décès ${ }^{3}$. D’après l'OMS, les accidents de la route sont la première cause de décès chez les 15 à 29 ans et, toujours pour ce groupe d'âge, les suicides se placent au deuxième rang et les homicides au quatrième rang ${ }^{3}$.

Au Canada, en 2010, les blessures ont coûté la vie à 16094 personnes et ont constitué la principale cause de décès entre 1 et $44 \mathrm{ans}^{4}$. Selon le Cost of Injury in Canada Report, et toujours en 2010 au Canada, les blessures ont fait peser un fardeau économique de 26,8 milliards de dollars, dont 15,9 milliards provenant des dépenses en soins de santé et 10,9 milliards de la baisse de productivité associée aux hospitalisations, aux invalidités et aux décès prématurés ${ }^{4}$.

Il est important de faire le suivi des causes et des modalités de décès, ainsi que de l'incidence des maladies et des blessures

\section{Points saillants}

- Pour comprendre les tendances et évaluer le fardeau en matière de blessures à l'échelle du Canada, il faut disposer de données nationales régulièrement mises à jour sur les décès attribuables aux blessures.

- En 2015, les blessures non intentionnelles ont constitué la sixième cause de décès en général et la principale cause de décès entre 1 et 34 ans. Le suicide a été la deuxième cause de décès chez les 15 à 34 ans.

- Les chutes, les empoisonnements et les accidents de la route ont constitué les trois principales causes de décès attribuables à des blessures non intentionnelles.

sur les individus, leurs familles, le système de soins de santé et la société dans son ensemble. Il est essentiel de fournir régulièrement des données nationales sur les décès attribuables à des blessures pour en comprendre les tendances, savoir à qui incombe le fardeau des blessures à l'échelle nationale et pouvoir élaborer des programmes ciblés de prévention des blessures. Dans cet article, nous présentons les statistiques canadiennes sur les décès attribuables à des blessures pour 2015 . Nous présentons aussi les principales causes de décès, ce qui permet de comparer les diverses catégories de blessures aux autres causes de mortalité, et donc de replacer le fardeau des blessures dans le contexte plus général de l'ensemble des causes de décès au Canada.

\section{Méthodologie}

Les données proviennent de la Base de données sur les décès $2015^{5}$ des statistiques 
de l'état civil de Statistique Canada. Les causes des décès ont été codifiées en suivant la Classification statistique internationale des maladies et des problèmes de santé connexes, dixième révision (CIM$10)^{6}$. Nous avons utilisé, comme dénominateur pour le calcul des taux, les estimations de population du Canada au $1^{\text {er }}$ juillet 2015 de Statistique Canada ${ }^{7}$.

Nous avons classifié et comparé les causes de l'ensemble des décès attribuables à des maladies et à des blessures en utilisant la CIM-10. Nous avons effectué des analyses plus approfondies sur les décès attribuables à des blessures en fonction de l'intention (non intentionnelle, suicide, homicide, intervention juridique ou guerre, ou intention non déterminée), en suivant là aussi la CIM-10. Nous avons comparé les causes externes de blessures (p. ex. empoisonnement, suffocation) mais en excluant les complications liées aux soins médicaux et chirurgicaux, dont la nature et les mesures de prévention sont différentes de la plupart des blessures ${ }^{2}$.

Nous avons effectué des analyses de données regroupées et stratifiées (par sexe et par âge). Pour compiler les effectifs et calculer les taux, nous avons utilisé SAS Enterprise Guide, version 5.1 ${ }^{8}$.

\section{Résultats}

Dans l'ensemble de notre étude, les taux sont calculés pour 100000 habitants et les taux normalisés selon l'âge (TNA) sont basés sur la population canadienne de 2015, hommes et femmes combinés.

\section{Principales causes de décès (ensemble)}

Le tableau 1 présente les effectifs et les taux de décès se rapportant aux principales causes de l'ensemble des décès. Le cancer et les maladies des appareils circulatoire et respiratoire sont les trois principales causes de décès chez les hommes comme chez les femmes.

Les blessures non intentionnelles sont à la sixième place de l'ensemble des causes de décès $(\mathrm{n}=11$ 833; taux $=33,0)$, plus spécifiquement à la cinquième place chez les hommes et à la septième chez les femmes. Le taux de mortalité attribuable à des blessures non intentionnelles est significativement plus élevé chez les hommes $(38,8$; intervalle de confiance [IC] à $95 \%$ : $37,9$ à 39,7$)$ que chez les femmes $(27,4$; IC à $95 \%$ : 26,6 à 28,1). La différence est encore plus grande si l'on examine les TNA (43,3; IC à $95 \%: 42,2$ à 44,3 contre 23,6; IC à $95 \%: 23,0$ à 24,3). Au sein de tous les groupes d'âge, les blessures non intentionnelles se positionnent parmi les huit premières causes. Elles constituent la principale cause de décès entre 1 et 34 ans, la deuxième cause de décès chez les 35 à 44 ans et la troisième chez les enfants de moins d'un an ainsi que chez les 45 à 64 ans.

Le suicide se place au $11^{\mathrm{e}}$ rang des causes de l'ensemble des décès ainsi que chez les femmes, mais au $9^{\mathrm{e}}$ rang chez les hommes. Il constitue l'une des principales causes de décès chez les jeunes et les jeunes adultes : au $2^{\mathrm{e}}$ rang chez les 15 à 34 ans et au $3^{\mathrm{e}}$ rang chez les 10 à 14 ans ainsi que chez les 35 à 44 ans. Chez les invidus plus âgés (45 à 64 ans), il se place au $6^{\mathrm{e}}$ rang en importance.

Les homicides se classent parmi les dix principales causes de décès chez les jeunes Canadiens de moins de 35 ans : au $4^{\mathrm{e}}$ rang chez les 20 à 24 ans, au $5^{\mathrm{e}}$ rang chez les 15 à 19 ans et au $6^{\mathrm{e}}$ rang chez les 25 à 34 ans.

\section{Principales causes de décès attribuables à des blessures}

Le tableau 2 présente le nombre et le taux de décès associés à des blessures en fonction de l'intention et fournit des détails sur les principales causes de blessures non intentionnelles.

En 2015, 17371 décès (taux $=$ 48,5) sont associés à des blessures : 10957 chez les hommes et 6414 chez les femmes. Les blessures non intentionnelles forment $68,1 \%$ des décès associés à des blessures, suivies par le suicide $(25,4 \%)$. Les principales causes de décès parmi les blessures non intentionnelles sont les chutes (39,8\%), l'empoisonnement $(19,9 \%)$ et les accidents de la route $(15,8 \%)$, suivis de la suffocation, de la noyade, du feu ou des flammes et enfin des heurts par ou contre un objet.

Les hommes ont un taux global de mortalité par blessure plus élevé $(61,7$; IC à $95 \%: 60,5$ à 62,8$)$ que les femmes $(35,5$; IC à $95 \%: 34,6$ à 36,4). On observe des taux de mortalité par blessure non intentionnelle plus élevés chez les hommes que chez les femmes pour toutes les causes principales sauf les chutes. Les TNA associés aux chutes sont cependant plus élevés chez les hommes $(15,7$; IC à $95 \%$ : 15,0 à 16,4) que chez les femmes $(11,3$; IC à $95 \%$ : 10,8 à 11,7). Les hommes et les femmes partagent le même classement pour les principales causes de décès non intentionnels, à l'exception de la noyade et du feu ou des flammes, mais, lorsque le classement est réalisé par groupe d'âge, on observe des variations. Les principales causes de décès sont la suffocation chez les moins d'un an, les accidents de la route chez les 1 à 24 ans et les empoisonnements chez les 25 à 64 ans. Chez les 65 ans et plus, les chutes correspondent à 64,6\% des décès attribuables à des blessures non intentionnelles. Le suicide est à l'origine de plus de décès que toute cause de blessure non intentionnelle chez les 10 à 64 ans.

\section{Analyse}

Les blessures ont un impact considérable sur les individus, les familles et les sociétés. Cet article fournit des statistiques nationales présentées par sexe et par groupe d'âge sur les décès attribuables à des blessures, ce qui est indispensable pour comprendre leur gravité et leurs tendances et prendre à partir de là des initiatives de prévention efficaces.

En 2015, les blessures non intentionnelles comptent parmi les dix principales causes de décès au Canada, chez les hommes comme chez les femmes et dans tous les groupes d'âge (tableau 1). Les hommes ont un taux de mortalité significativement plus élevé que les femmes pour les blessures non intentionnelles. La différence entre les TNA est encore plus importante. Les hommes affichent des taux de mortalité plus élevés que les femmes pour toutes les causes principales de décès à l'exception des chutes. Les TNA associés aux chutes montrent cependant que les hommes dépassent là aussi les femmes. Cela s'explique principalement par le fait qu'il y a plus de femmes que d'hommes de plus de 60 ans au Canada et que le taux de mortalité associé aux chutes augmente considérablement au sein de la population âgée. Un examen détaillé a révélé que les taux de mortalité associés aux chutes sont plus élevés chez les hommes que chez les femmes dans presque tous les groupes d'âge de cinq ans. Si l'on examine les différences d'âge, les catégories plus âgées ont des taux de mortalité beaucoup plus élevés à la suite de blessures non intentionnelles que les groupes plus jeunes, mais à 


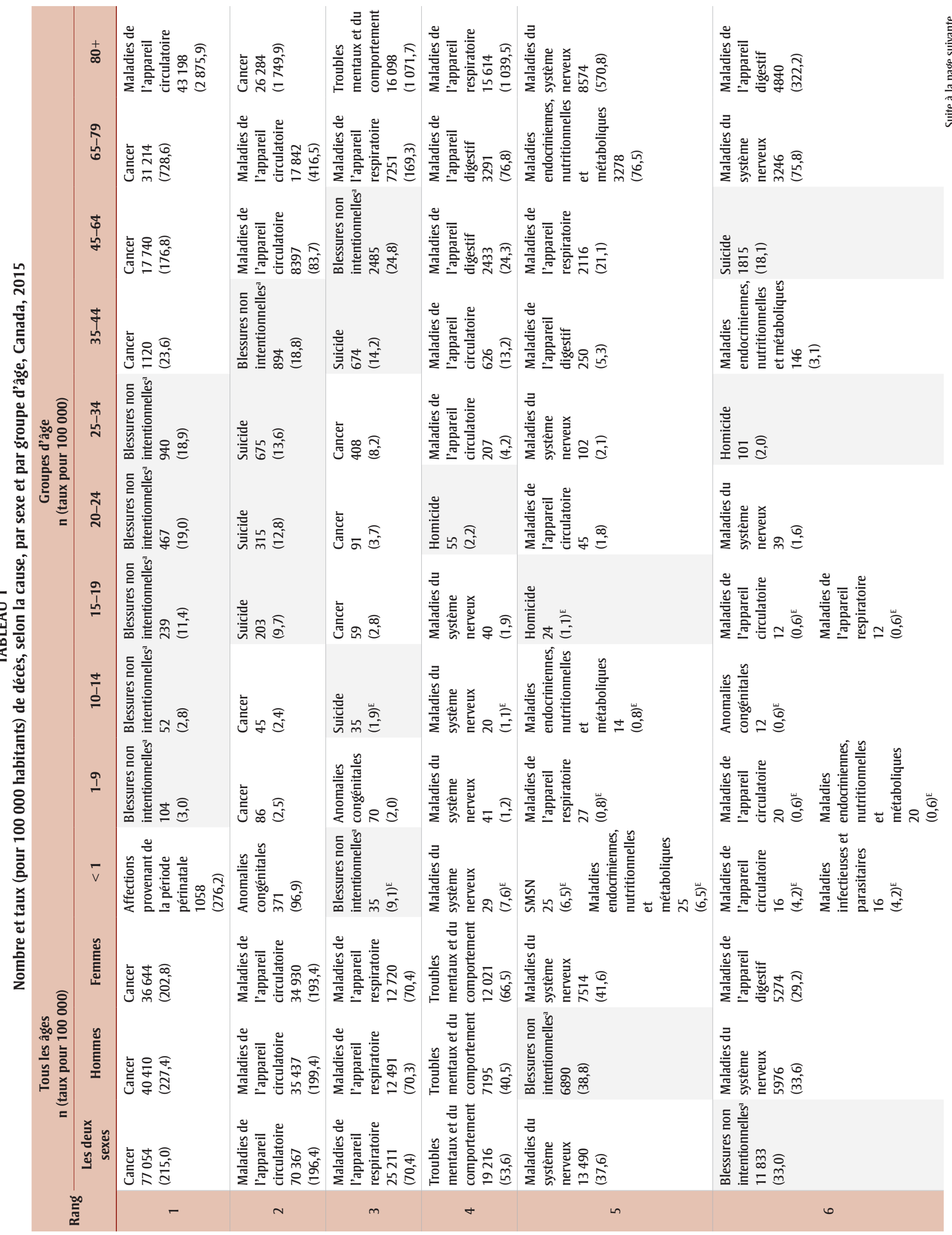




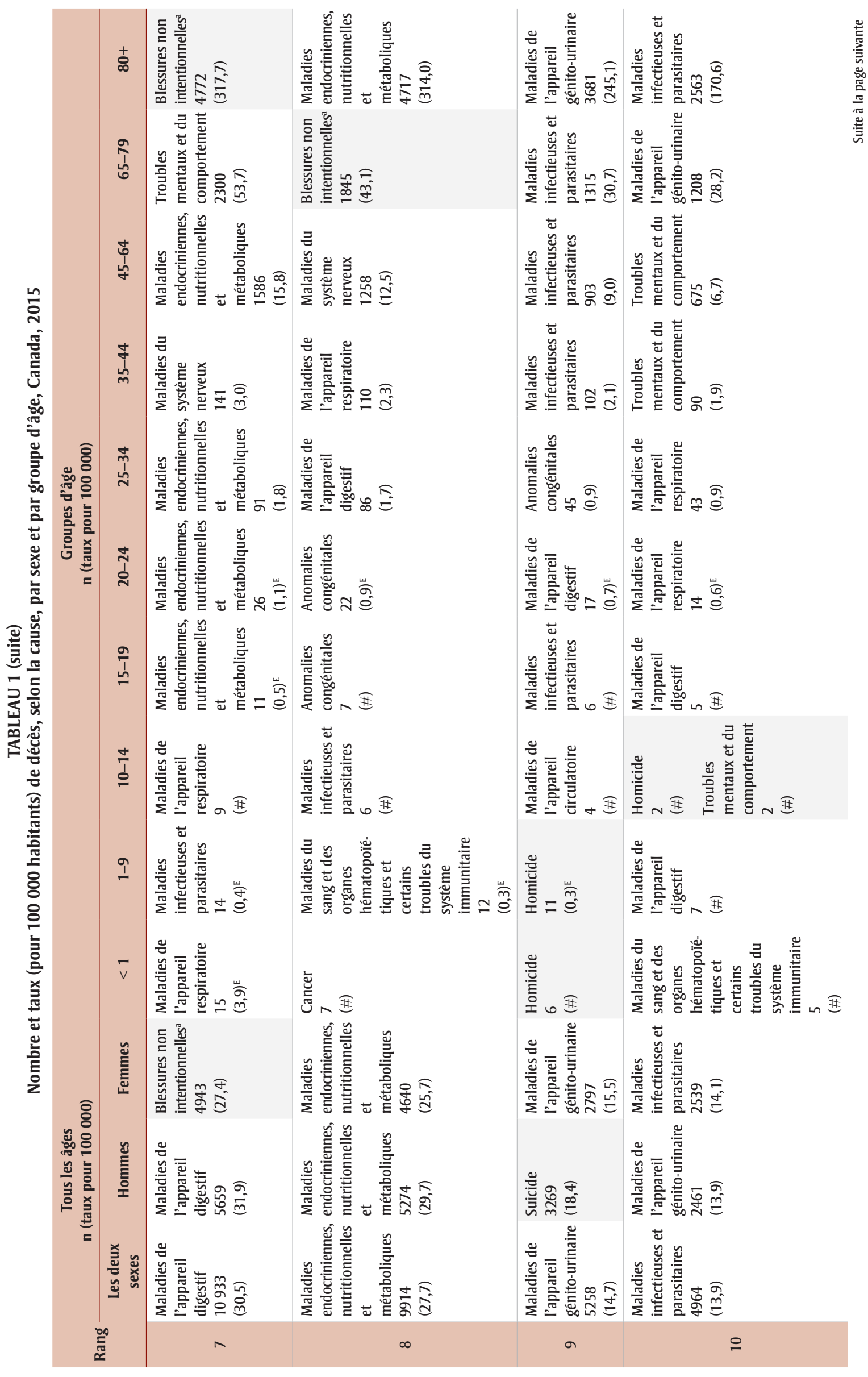




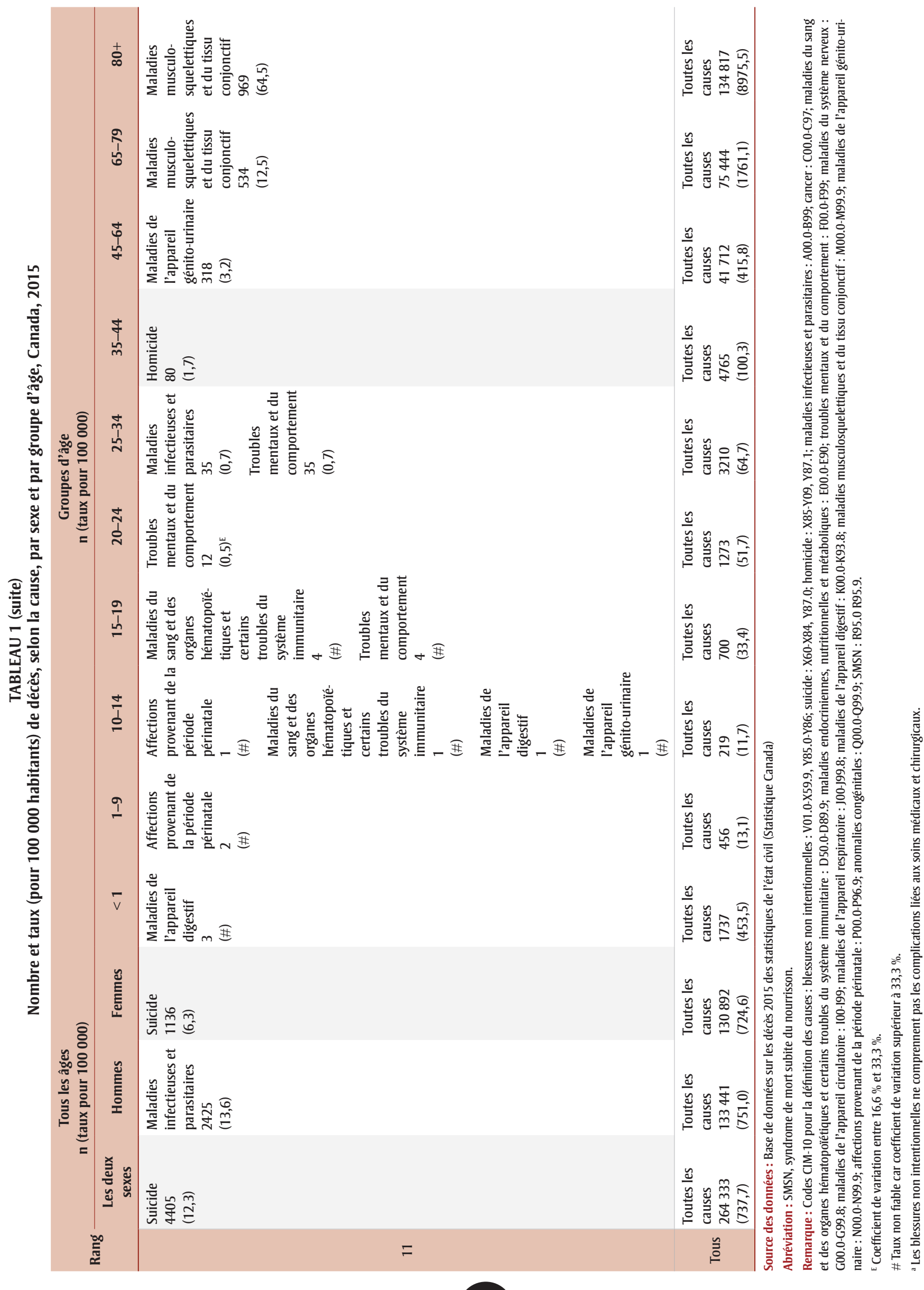


TABLEAU 2

Nombre et taux (pour 100000 habitants) de décès attribuables à des blessures, selon la cause, par sexe et par groupe d'âge, Canada, 2015

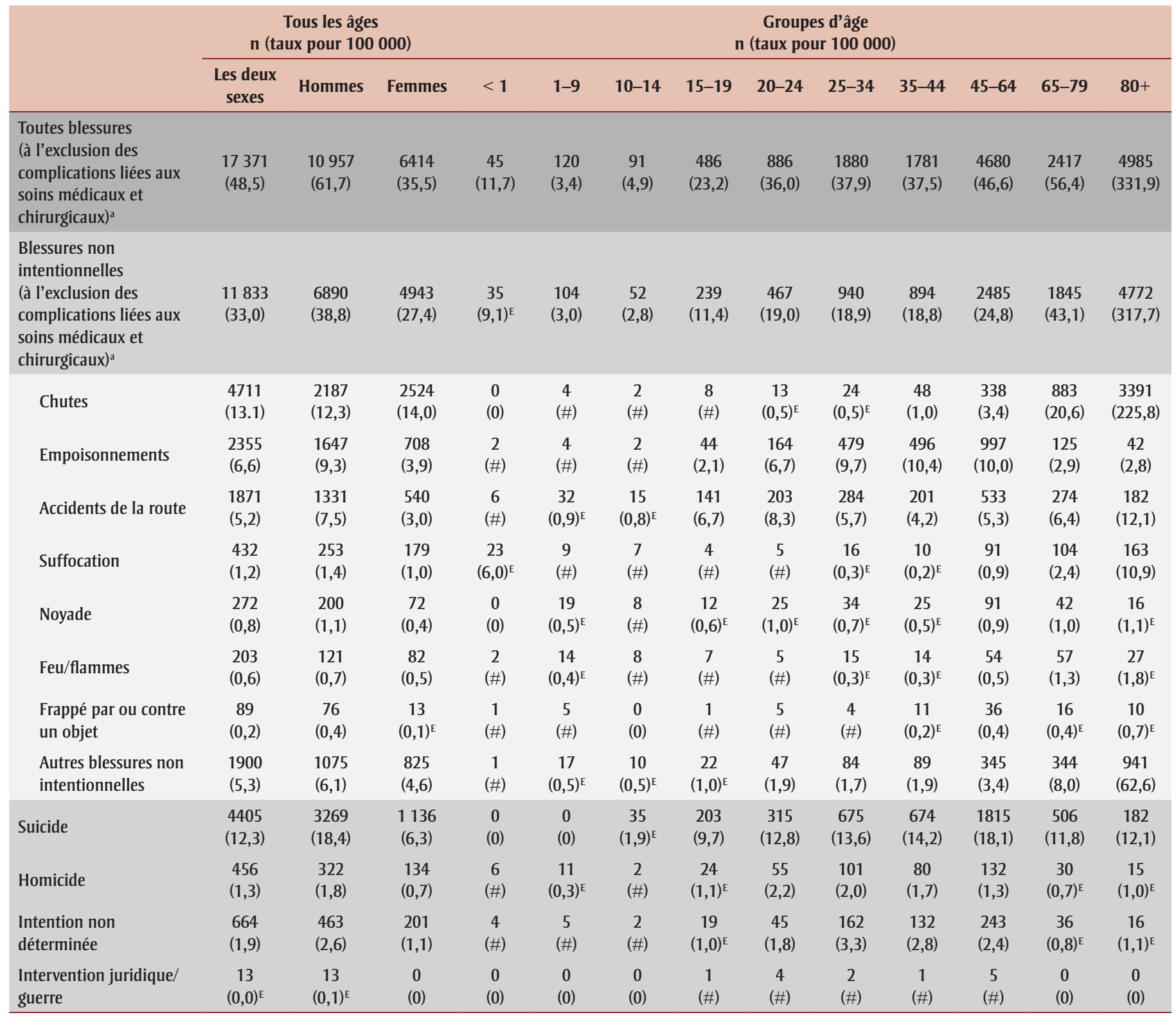

Source des données : Base de données sur les décès 2015 des statistiques de l'état civil (Statistique Canada)

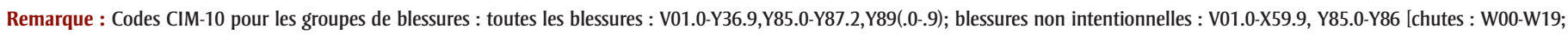

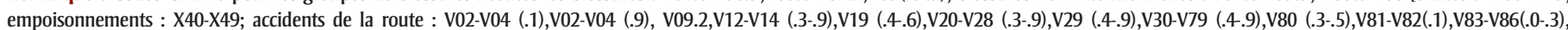

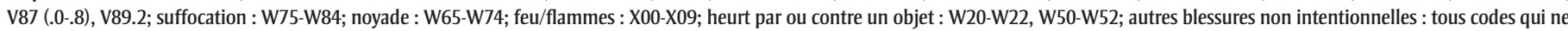

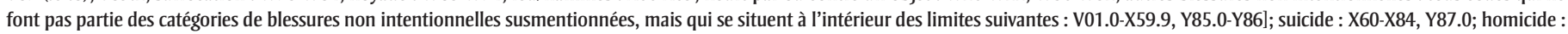
X85-Y09, Y87.1; intention non déterminée : Y10-Y34, Y87.2, Y89.9; intervention juridique/guerre : Y35.0-Y36.9,Y89(.0-.1).

${ }^{\mathrm{E}}$ Coefficient de variation du taux entre $16,6 \%$ et $33,3 \%$.

\# Taux non fiable car coefficient de variation supérieur à 33,3\%.

${ }^{a}$ Codes CIM-10 pour les complications liées aux soins médicaux et chirurgicaux : Y40-Y84, Y88. 
un rang inférieur à celui des groupes plus jeunes en raison de l'augmentation des maladies chroniques. Cela indique que les stratégies de prévention devraient varier en fonction des groupes d'âge.

Il est à noter que les empoisonnements se sont hissés à la deuxième place des causes de décès par blessure non intentionnelle (tableau 2). Avant 2014, c'étaient les accidents de la route qui occupaient cette place. D’autres recherches sont nécessaires pour comprendre le rôle que jouent, dans cette augmentation des empoisonnements non intentionnels, les opioïdes et autres drogues illicites 9 .

En 2015, le suicide s'est classé au deuxième rang des causes de décès chez les 15 à 34 ans, derrière les blessures non intentionnelles (tableau 1). Cependant, l'examen des causes externes parmi les blessures non intentionnelles (tableau 2) a révélé que les suicides dépassaient les décès non intentionnels par accident de la route au sein de ce groupe d'âge (15 à 34 ans). Cela souligne la nécessité d'une prévention efficace du suicide au sein de cette population.

\section{Limites}

La base canadienne de données sur les décès des statistiques de l'état civil ne fait état que de la cause sous-jacente du décès qui déclenche la suite d'événements menant directement au décès ${ }^{10}$ et ne tient pas compte des autres blessures qui peuvent avoir joué un rôle dans le décès, ce qui pourrait entraîner une sous-déclaration de la mortalité liée aux blessures.

Cet article a pour but de fournir une information importante et de qualité permettant aux intervenants d'évaluer le fardeau des blessures par rapport à d'autres causes de décès dans le cadre des programmes de prévention des blessures. Les informations seront mises à jour lorsque de nouvelles données sur la mortalité seront disponibles.

\section{Conclusion}

Les blessures demeurent l'une des principales causes de décès au Canada en 2015. Dans l'ensemble, les hommes courent un plus grand risque de décès attribuables à des blessures non intentionnelles que les femmes. Néanmoins, les principales causes de décès attribuables à des blessures non intentionnelles varient tout au long de la vie. Les blessures non intentionnelles, le suicide et les homicides sont responsables de nombreux décès chez les jeunes Canadiens. Cet article fournit des données utiles pour évaluer le fardeau des blessures et comprendre les tendances.

\section{Conflits d'intérêts}

Les auteurs déclarent n'avoir aucun conflit d'intérêts.

\section{Contributions des auteurs et avis}

Tous les auteurs ont lu et approuvé le contenu de l'article. XY a participé à la conception, à l'analyse et à l'interprétation des données ainsi qu'à la préparation du manuscrit. RS, SM et WT ont participé à la conception et à l'interprétation des données ainsi qu’à la préparation du manuscrit.

Le contenu de l'article et les points de vue qui y sont exprimés appartiennent aux auteurs et ne reflètent pas nécessairement ceux du gouvernement du Canada.

\section{Références}

1. Robertson, L.S. Injury epidemiology: research and control strategies. 3e éd. New York (NY) : Oxford University Press; 2007.

2. Agence de la santé publique du Canada. Étude des blessures, édition 2012 : Pleins feux sur la sécurité routière en matière de transport. Ottawa (Ontario) : Agence de la santé publique du Canada; 2012.

3. Organisation mondiale de la Santé (OMS). Traumatismes et violence : Les faits 2014 [Internet]. Genève (Suisse) : OMS, 2014. En ligne à : https://apps .who.int/iris/handle/10665/161367

4. Parachute. The Cost of Injury in Canada Report [Internet]. Toronto (Ontario) : Parachute; 2015 [consulté le 3 déc. 2018]. En ligne à : http:// www.parachutecanada.org/costofinjury

5. Statistique Canada. Statistique de l'état civil - base de données sur les décès 2015. Ottawa (Ontario) : Statistique Canada; 2017.

6. Organisation mondiale de la Santé (OMS). International Statistical Classification of Diseases and Related Health Problems, Tenth Revision [Internet]. Genève (Suisse) : OMS; 2016 [consulté le 3 déc. 2018]. En ligne à : https:// icd.who.int/browse10/2016/en
7. Statistique Canada. Canadian population estimates by age, sex, and provinces and territories for October 1 , 2017 [ensemble de données interne de Statistique Canada]. Ottawa (Ontario) : Statistique Canada; 2017.

8. SAS Institute Inc. SAS Enterprise Guide 5.1. Cary, NC, É.-U.

9. Santé Canada. Opioïdes et crise des opioïdes - Obtenez les faits [Internet]. Ottawa (Ont.) : Santé Canada; [modifié le 11 janv. 2019]. En ligne à : https://www.canada.ca/fr/sante -canada/services/dependance-aux -drogues/consommation-problematique -medicaments-ordonnance/opioides /obtenez-les-faits.html

10. Division de la statistique des Nations Unies. Principles and Recommendations for a Vital Statistics System. Revision 3. New York (NY) : ONU; 2014. 